\title{
(Free) Single Sign On Solution
}

\section{Graham McCarthy gmccarthy@ryerson.ca Library Systems Analyst Ryerson University Library}




\section{$\mathrm{Hmm}$. free?}

What's the catch...

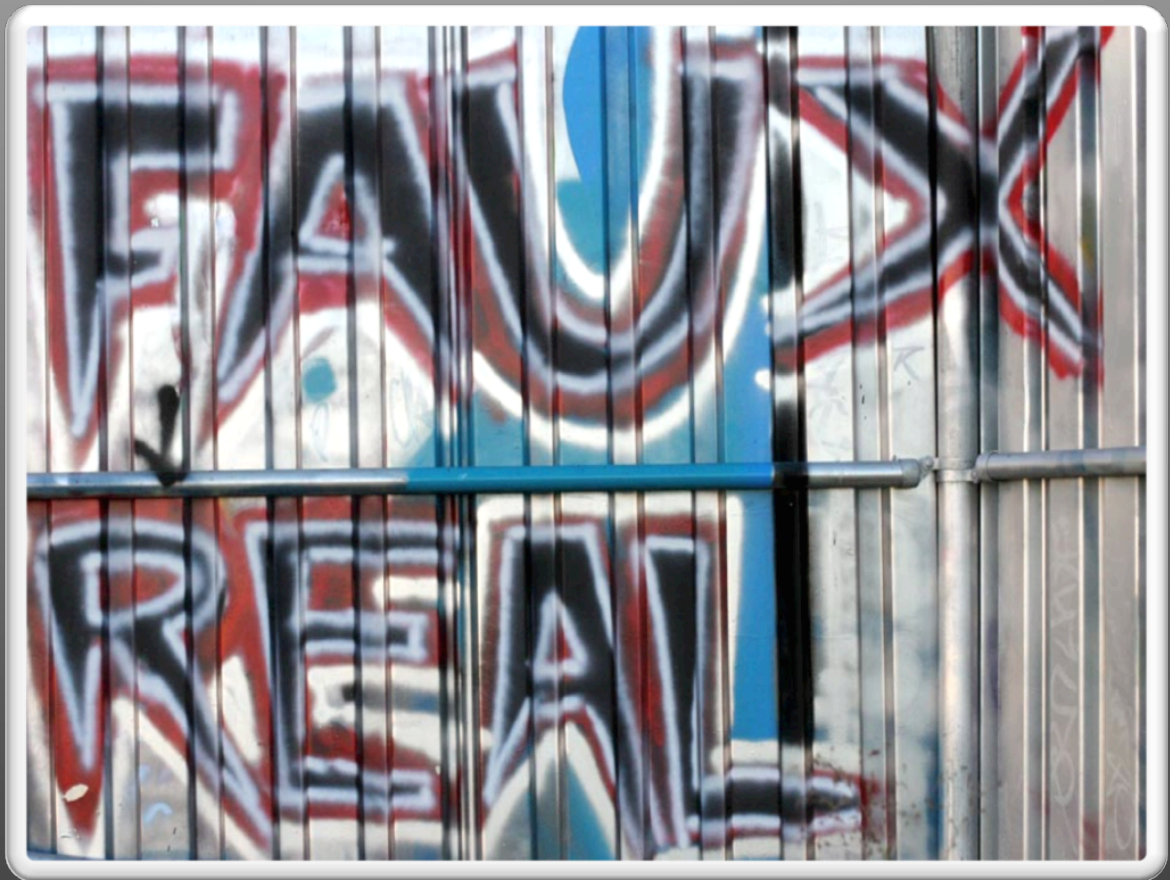




\section{Faux?}

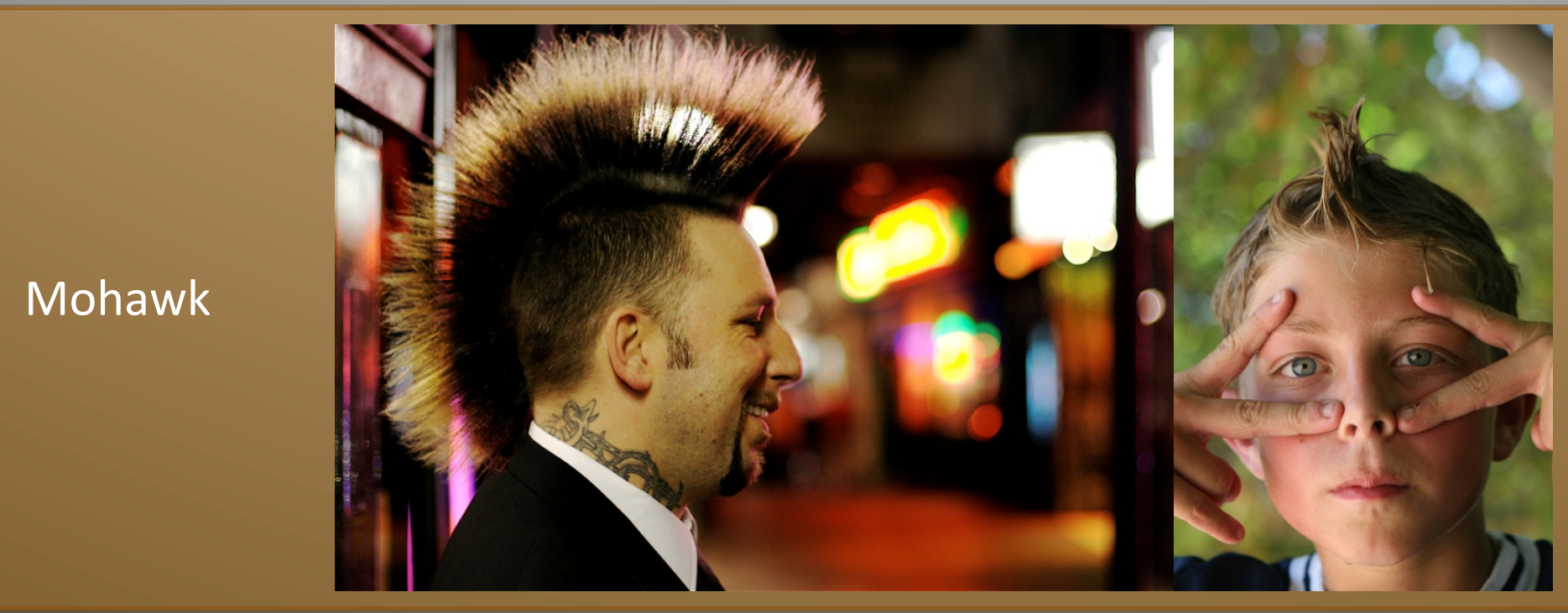

Faux-Hawk

Faux (pronounced /for/, like "foe") is a French word for false or fake. It is often used in English phrases such as faux pearls, faux fur, faux pas and faux news. 


\section{Faux Sign On}






\section{What have you been doing?}

Academic:

\begin{tabular}{|l|l|l|l|}
\hline Institution & Single Sign On & Faux Sign On & Barcode/Pin/Name \\
\hline Western & & & \\
\hline Brock & & & \\
\hline Ryerson & & & \\
\hline Carleton & & & \\
\hline Ottawa & & & \\
\hline Concordia & & & \\
\hline
\end{tabular}

Public:

\begin{tabular}{|l|l|l|l|}
\hline Institution & Single Sign On & Faux Sign On & Barcode/Pin/Name \\
\hline Cambridge & & & \\
\hline Kitchener & & & \\
\hline London & & & \\
\hline $\begin{array}{l}\text { Thunder Bay } \\
\text { Waterloo }\end{array}$ & & & \\
\hline
\end{tabular}

Government:

HRSDC : ? 


\section{Down to business...}

\section{Using your One Card Information}

Traditional:

(located on Innovative

Server)

\author{
Your Name: \\ (either first or last name) \\ Your 13 digit barcode: \\ (on your staff/student card) \\ Your Library PIN: \\ (last 4 digits of student/staff number)
}

\section{Database diagram:}

Campus Username

Library Barcode

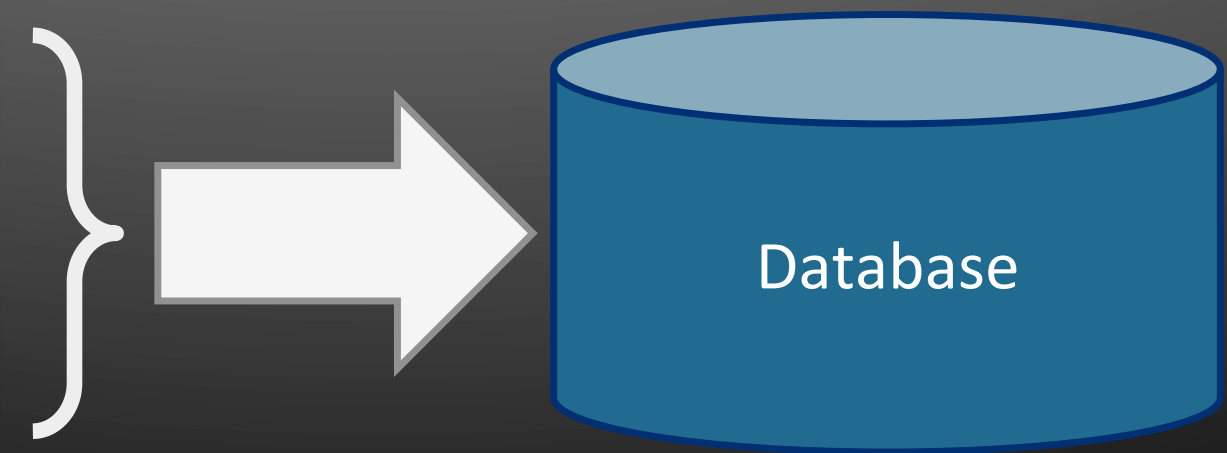




\section{Check Your File}

\section{Ryerson login page:} (located on Innovative Server)

\section{Using your my.ryerson Account}

(what are the benefits?)

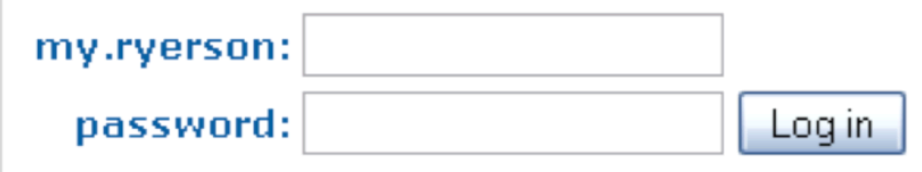

Requests

Authentication

Library

Server
Campus

LDAP server
Authenticated

Session 


\section{System Design Cont'd ...}

\section{Welcome, Graham!}

Enter PIN page:

(located on a Library Server)
Please enter your library PIN to sign into your borrower record.

\section{Library PIN:}

View your borrower record
Enter Pin Page
Sends Barcode, Username and PIN
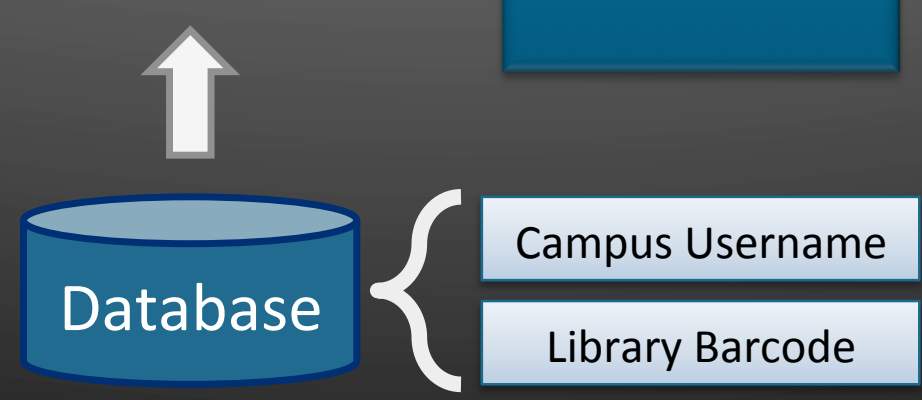

Innovative Server (Login Page) 


\section{Opt In Service}

\section{Welcome, Graham!}

First login with campus ID

\section{(located on a Library Server)}

Since this is your first time logging in to your borrower record this way, we need to store your 13 digit barcode.

\section{(Why not store my library PIN as well?)}

Once completed, you won't need to provide this information ever again!

We need your 13 digit barcode:

(Where do I find this?)

Submit Information

Barcodes are verified by using a MOD10 Check Algorithm

(Similar to how Credit Cards are validated) 


\section{What about placing holds?}

Holds page:

(On Innovative Server)

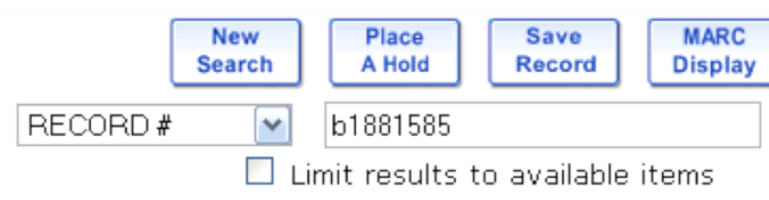

Search

Title Intertwinings : interdisciplinary encounters with Merleau-Ponty / edited by Gail Weiss.

Publisher Albany: State University of New Vork Press, c2008.

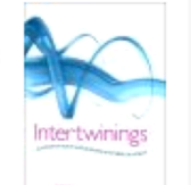

\section{Place a Hold}

Requesting Intertwinings : interdisciplinary encounters with Merleau-Ponty / edited by Gail Weiss.

\section{Login to place} holds page:

(On Innovative Server)

\section{Using your my.ryerson Account}

(what are the benefits?)

my.ryerson:
password:

\section{Authentication and Redirection (On Library Server)}

\section{Request On Hold:} (On Innovative Server)

\section{RECORD \#}

\section{b1881585}

$\square$ Limit results to available items

\section{Search}

Vour request for Intertwinings : interdisciplinary encounters with Merleau-Ponty / edited by Gail Weiss. was successful. 
1. Ability to modify the pverify_web.html

2

Access to a web server which can connect to an LDAP server

Also: TLS/SSL encryption enabled

3 Ability to create a database
Brief Legend:
LDAP = lightweight directory access protocol
- used for authenticating users
TSL = Transport Layer Security
SSL = Secure Sockets Layer
TSL and SSL are used to encrypt data through transfer.


Data passing between servers is sensitive

You need to protect your patrons information

How do you know?

- Look for " https://" in the URL

- Look for the closed yellow lock at the bottom of your browser.

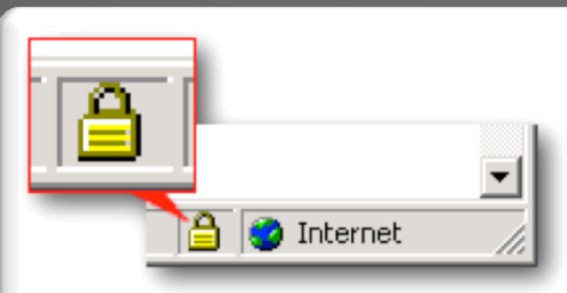


Cookie (pronounced ' $\backslash k u$-kē $\backslash^{\prime}$ ) is a a small flat or slightly raised cake.

2. An attractive woman

3. A small file stored on a user's computer containing identifying information read by a web server (Definition from http://www.merriam-webster.com/dictionary/cookie) 


\section{Problems with the Faux Sign On}

AirPac

- Does not allow edits to the login page.

Millenium Updates

- Cross fingers that Innovative do not change the way they perform patron logins. (So far so good!) 


\section{Usage Statistics}

\section{Statistics as of (April $1^{\text {st }}$ 2009)}

Patrons with active library accounts: 34,111

Patrons who have checked out at least one item: 23,899

Patrons using the Faux Sign On: 10,166

Patrons who stopped using Faux Sign On: 3

$42.5 \%$ usage of active library users who check out material

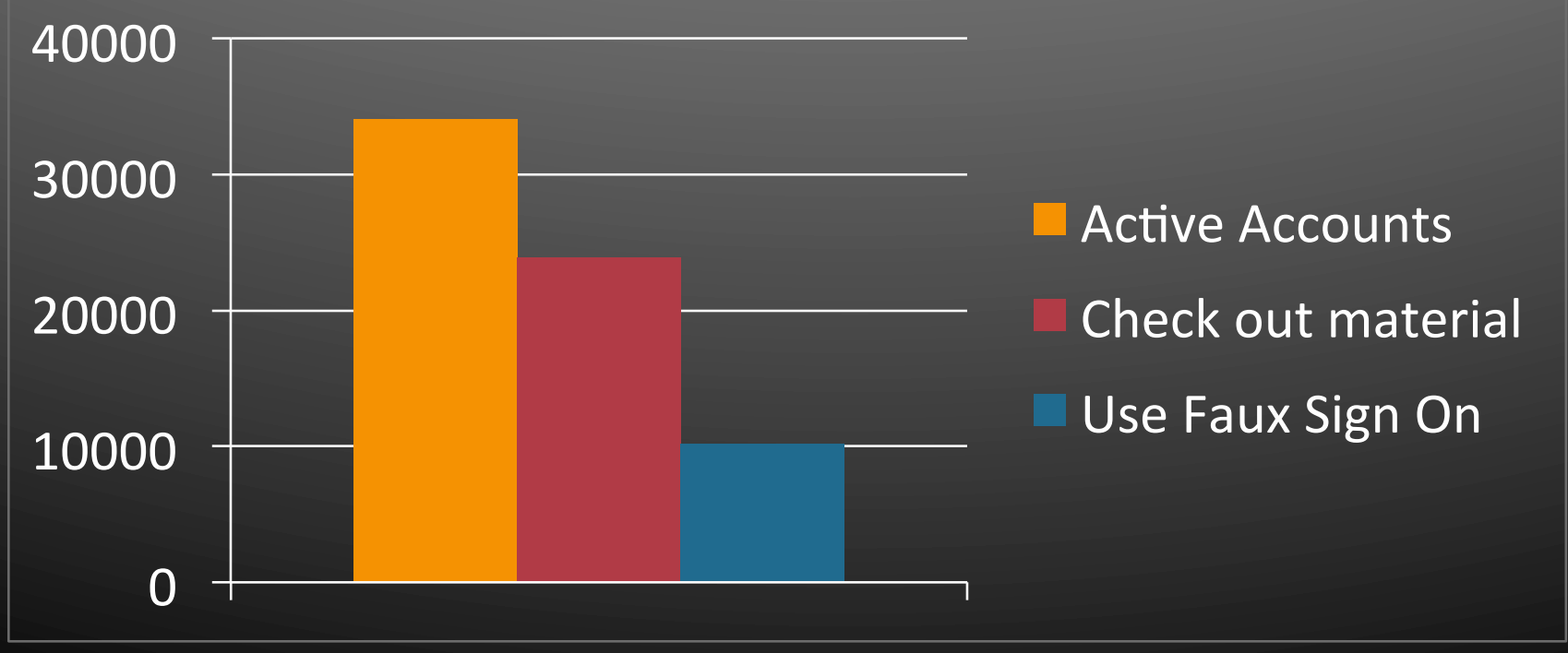




\section{Stats as of (April 1st 2009)}

Total usage since release (Sept $2^{\text {nd }} 2008$ ): 72,603

Students: $9,681 \quad(95.0 \%)$

Faculty: $\quad 363 \quad(4.0 \%)$

Staff: $\quad 109 \quad(1.0 \%)$

Other: $\quad 16 \quad(0.0 \%)$ Approx.

\section{Usage Ratios}

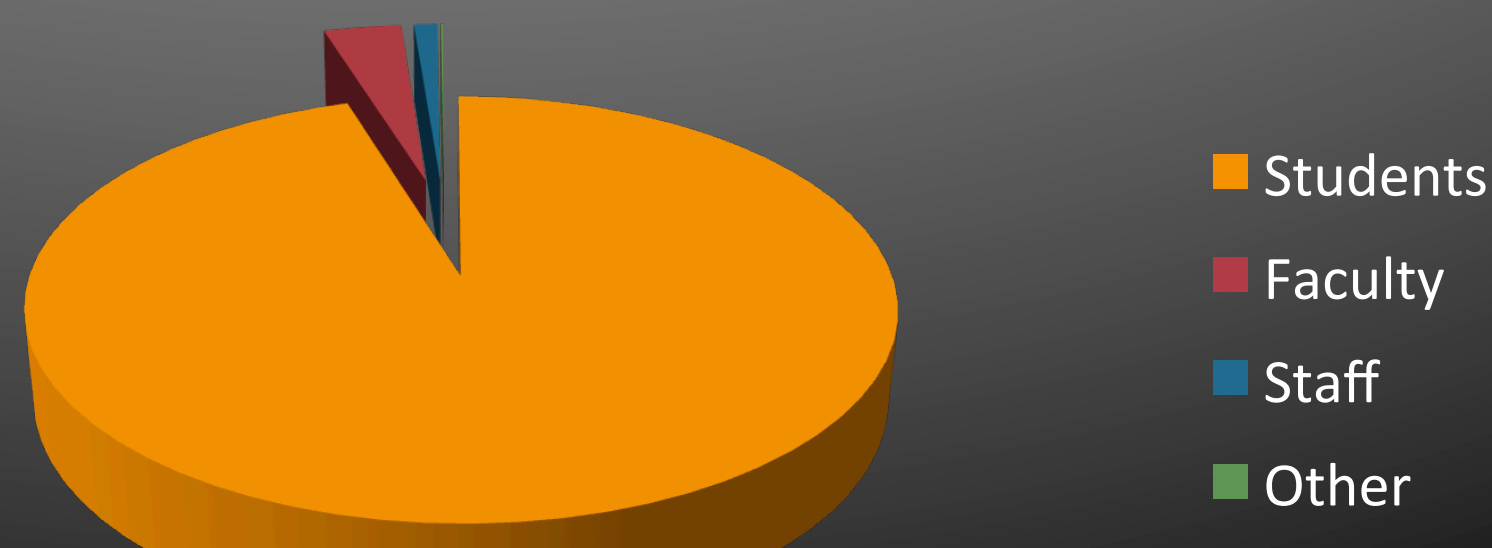




\section{Faux Sign On is More than a Campus Login}

\section{facebook.}

Login to library account:

- Through a Facebook application

- Using an e-mail address instead of a barcode

Windows Live Hotmail.

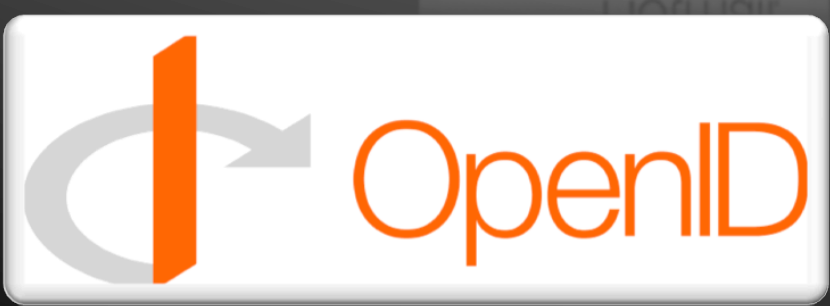

- Using a Patron's OpenID 


\section{Contact Information}

Graham McCarthy

gmccarthy@ryerson.ca

$416.979 .5000 \times 2119$

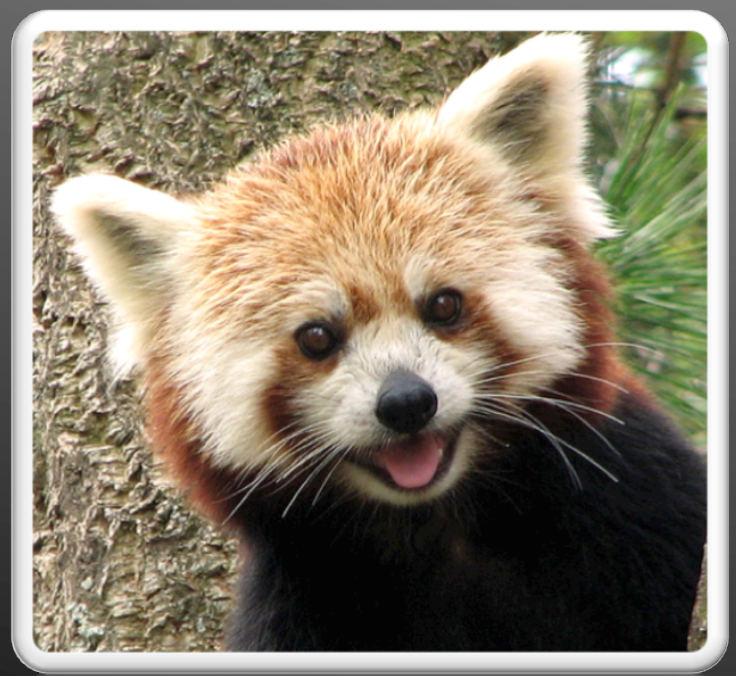

Ideas

Sharing... Designs

Experiences

Code 


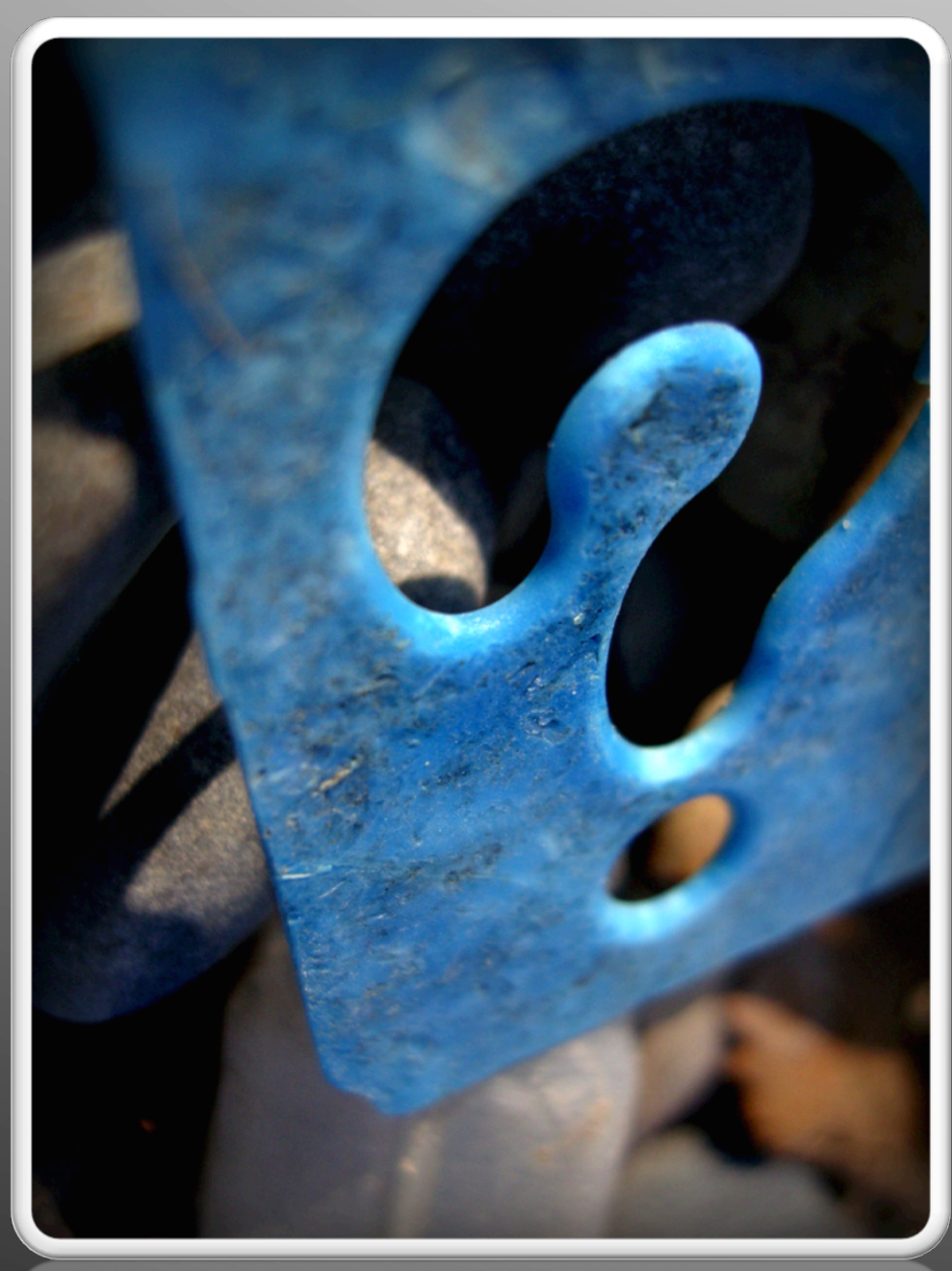

\section{Questions?}

\section{Flickr Acknowledgements:}

Graffiti - http://www.flickr.com/photos/gwen/3148081279/sizes/o/ Mohawk - http://www.flickr.com/photos/tommyforbes/385283613/sizes/I/ Faux-Hawk - http://www.flickr.com/photos/davidden/1763265904/sizes/l/ Hippos - http://www.flickr.com/photos/22193699@N04/3040692147/sizes/o/ Lamma - http://www.flickr.com/photos/from_linda_yvonne/3315408178/ Cat - http://www.flickr.com/photos/26686573@N00/1105321884/

Fuzzy Thing - http://www.flickr.com/photos/fogassa/2218047791/

Cookie Monster - http://www.flickr.com/photos/esti/147733640/sizes/l/ (Fuzzy Thing again)

Question Mark - http://www.flickr.com/photos/87765855@N00/3105128025/sizes/I/ All images licensed under Creative Commons 